\title{
Playing it by ear: Overcoming the limitations of speech synthesizers
}

\author{
EMERSON FOULKE \\ University of Louisville, Louisville, Kentucky
}

\begin{abstract}
Inexpensive speech synthesizers are now available that either plug into the card slots on computers or connect to their serial ports. With some practice in listening, the speech produced by these synthesizers is quite intelligible, and because they present information that appears on the screen of the monitor, they make it possible for blind persons to interact with computers. However, they provide only a partial solution. In too many instances, some of the text appearing on the monitor screen cannot be directed to the synthesizer, and the missing information is often crucial. This happens because the writers of application programs sometimes modify the computer's disk operating system. This limitation can be overcome by using special hardware that reads the screen buffer directly and creates a virtual image of the screen in memory external to the computer. Because the image is updated continually, it is always an accurate representation of whatever appears on the screen. The characters contained in the external buffer can be sent to a speech synthesizer, and can be examined selectively by means of a small keyboard. Several successful hardware solutions have been demonstrated, and one of these solutions is described here in detail.
\end{abstract}

Far too many blind people are either underemployed or unemployed, and their inability to read and write print, and to interact comfortably with those who do, must be recognized as a major impediment to the realization of human potential. An effective solution to this unemployment problem will not be possible until a practical way of reducing the print handicap is implemented.

For 30 years and more, it has been obvious to many that, by taking advantage of the flexibility of computers in regard to the display of text (print, braille, synthetic speech), blind workers could largely overcome their print handicap. However, until recently, the solution offered by the computer was impractical: because the systems (minicomputers or mainframes) were too expensive for individuals to own, and because the number of such computers was comparatively small, few blind people had or would ever have access to them.

With the introduction of microcomputers, the situation has changed dramatically (Foulke, 1982). The availability of inexpensive and powerful microcomputers has brought about a major revision of the way we perform tasks at work, at school, and at home, and because tactual and auditory alternatives to the visual display of information are now available, blind people can also use computers. Though they are expensive in comparison to the printers used by sighted computer operators, braillers, which perform equivalent functions, are available from several sources. Devices with transitory braille displays, like the transitory print displays on the screens of monitors, are also available. However, they are very expen-

Reprint requests should be sent to Emerson Foulke, Perceptual Alternatives Laboratory, University of Louisville, Louisville, KY 40292. sive, and to prevent their prices from becoming altogether unrealistic, it has been necessary to limit severely the amount of text they can display at one time. For example, one of the most widely used and successful devices of this type can display only a single line of 20 characters at one time (Melrose, 1984).

In the past few years, the technology for the synthesis of speech has expanded dramatically in terms of both the implementation of effective techniques and the reduction of costs. It is now possible to buy speech synthesizers, which are either connected to the ports of microcomputers or plugged directly into their card slots, at prices from $\$ 100$ to $\$ 300$ (Lowry, 1985; Scadden, 1983). Although the speech generated by these inexpensive synthesizers may initially be difficult to understand, with practice in listening it quickly becomes intelligible, and particularly so if the person who must understand the speech is also the computer operator who has entered the text of the speech on the keyboard of the computer.

Even inexpensive synthesizers can render words with impressive fidelity if they are required only to reproduce a restricted vocabulary in which the phonemes needed for each word and the proper amplitudes, durations, and pauses have been carefully selected. However, to support the interaction of a blind operator and a computer, a synthesizer must be capable of displaying an unlimited vocabulary. This capability is provided by a text-to-speech program, which examines text stored in computer memory and uses rules to replace the letters in words with the phonemes required for their pronunciation. Because English is a notoriously irregular language, it is not possible to devise a set of rules that will select the correct replacements on every occasion, and as a result, words are often mispronounced. This problem can be mitigated by the use 
of an exceptions table, but it is not eliminated, and listeners must adapt to an aural display in which some words are mispronounced. However, blind computer operators quickly adjust to the idiosyncrasies of their synthesizers.

For a synthesized speech display to be useful to a blind computer operator, the operator must be able to do much more than just listen, from top to bottom, to the aural counterpart of the visual display. Just as the sighted operator can look selectively at any part of the visual display, the blind operator must be able to listen selectively to any part of the aural display. This is a difficult requirement to meet because, whereas the entire visual display is continually available for observation, the aural display unfolds temporally, and the only part of it that is available for observation at any instant is the speech generated by the synthesizer at that instant. The blind computer operator must resort to keyboard commands to move to the part of the display where needed information is to be found. For this screen navigation to be useful, movement through the display must be quick and precise. The operator must be able to move from the top to the bottom of the display, or vice versa, with a single command, and to choose any specific line or position within a line by issuing appropriate keyboard commands. Once the desired location in a display has been reached, the operator must be provided with commands that present text a character at a time, a word at a time, a sentence at a time, and so forth. Other commands must be available that make it possible to retrace in the same way.

Several word processors are now available for the Apple computer that have been specially designed to facilitate interaction with the computer by means of selective access to a synthesized speech display (Croft, 1986; Espinola, 1985; McGillivray \& Gast, 1983). These programs are generally satisfactory, but they share a major problem. The commands they provide for use by the operator are effective only within the word processing programs. When the operator leaves any of these programs, the computer no longer understands the commands.

Several programs are available for computers of the IBM PC type that aim to provide review of the screen by means of synthesized speech for whatever program is currently running (Croft, 1984; Espinola, 1985). To the extent this objective is realized, the blind operator is able to use any available software, instead of being restricted to the use of application programs specially designed for use by blind operators.

As a class, these programs are somewhat less convenient than the word processing programs just discussed. The operator who uses one of them is no longer using a single-application program, which, in addition to the customary commands for accomplishing the program's purpose, includes the special commands needed for the kind of screen navigation that makes synthesized speech useful. Instead, the operator is running two programs concurrently, the application program and a second program, which provides the commands needed for screen naviga- tion. Suppose that a blind computer operator is using a commercially available word processing program such as WORDSTAR, and is interacting with the computer by means of a screen review program. If the operator wishes to review information already entered, it is necessary to leave the word processing program temporarily and activate the screen review program, use the commands provided by the review program for the desired review, and then return to the word processing program. Although the screen review program allows the operator to determine the screen position of any character, it provides no commands for moving the cursor. Therefore, if review has revealed an error that must be corrected, the operator must determine and remember its screen position so that, on reentering the word processing program, he or she can move the cursor to that position, correct the error, and return the cursor to the appropriate position for the continuation of text entry. If the operator wants assurance that the correction has been adequate, it is necessary to leave the word processing program and enter the screen review program again, issue a command that will allow the operator to commence reading at the remembered position of the correction, and then leave the review program and return to the word processing program again.

To take another example, the operator who is using one of the word processing programs specialized for use by blind operators can, by issuing a keyboard command, listen to the announcement of all of the available menu choices. When the menu has been recited, the operator can resume work without having to issue a command to reenter the word processing program. The blind operator of a word processing program that has not been specialized for use by blind operators can issue the command provided by the word processing program for displaying menu options, and they will appear on the screen; but to read them, the operator must leave the word processing program and enter the screen review program, issue the commands provided by the screen review program for examining the screen, return to the word processing program, and issue the command that elicits the desired menu option.

These problems make screen review programs inconvenient to use, but this inconvenience diminishes as skill is acquired and is not a formidable factor for the experienced blind operator. However, there is another problem that does not diminish as skill is acquired. Like other programs, screen review programs use the computer's disk operating system to achieve their results, and since these programs must run on a variety of computers that use the same operating system, their designers must assume the availability of an operating system that has not been modified. However, for a variety of reasons, programmers sometimes decide to bypass the procedure provided by the operating system for routing information to the screen, and to use, instead, procedures they have devised to suit their purposes. To do this, they must modify the operating system, and when the screen review program encounters a modified operating system, it no 
longer functions. The operations on which it depends for getting information to the screen are no longer available. Consequently, blind computer operators sometimes find that they cannot interact with programs they would like to use, because the programs are not compatible with their screen review programs. Any screen review program could, of course, be modified to meet the demands of a particular application program, but, for obvious reasons, this is not a practical solution.

Another problem of the same type arises because the screen review program and the application program must inhabit the computer's memory at the same time. The screen review program's address in memory is chosen to minimize the likelihood that it will be competing with an application program for the same space in memory. However, such conflicts occasionally occur, and they render the screen review program ineffective. These conflicts can be resolved by a reallocation of memory, but this is not a practical solution.

The methods so far discussed for providing users of speech synthesizers the ability to review the screen have been implemented in software. When this approach is used, conflicts with application programs for the computer's operating system and for space in memory occur often enough to cause problems for blind computer operators. These problems can be avoided by implementing the method for reviewing the screen in hardware, and this is the approach that was taken by $T$. V. Cranmer, the Director of Research and Development for the National Federation of the Blind (NFB), and NFB's Research and Development (R\&D) Committee (Chong, 1986).

The R\&D Committee developed a card (the Speaqualizer) that plugs into a card slot of the IBM PC computer. It uses neither the computer's operating system nor its memory. Instead, it monitors the computer's screen buffer and captures in memory a continually updated image of the screen display. The card also contains a speech synthesizer, a text-to-speech program for operating the synthesizer, and the program that enables review of the screen image captured in its own memory. A cable connects the card to a box outside the computer, which contains a keyboard used for controlling screen review, a loudspeaker for the synthesizer, a headphone jack, and a port through which characters received by the Speaqualizer can be forwarded to a braille device.

The Speaqualizer's repertoire of keyboard commands includes commands for current letter, next letter, last letter, current word, next word, last word, current line, next line, last line, top of screen, bottom of screen, any specified line on the screen, screen position of current character, spelled speech, pronounced speech, and so forth. There are commands for changing the loudness and word rate of the speech, and a command for specifying a line of the screen to be monitored continually. When the monitoring function is engaged, error messages or other status changes displayed on this line interrupt any speech in progress, and the operator is notified of the change immediately.
The Speaqualizer provides instant screen review. It is not necessary to halt a running program in order to engage a screen review program. Because the Speaqualizer is a passive device that makes no use of the computer, there is never any competition for the computer's operating system or memory. Any information that is displayed on the screen is also in the Speaqualizer's screen image, and hence is available to the blind computer operator.

Although the Speaqualizer solves problems not solved by software approaches, it does not solve every problem. Graphic information displayed on the screen is faithfully captured in the Speaqualizer's memory. Of course, it is not rendered intelligibly by the speech synthesizer. The only solution is to suppress graphic information, so that the blind computer operator is spared the ordeal of listening to the noise it produces.

Another problem arises when programmers devise new screen attributes and substitute novel cursors for the system cursor. The Speaqualizer can be programmed to identify these features, but it is not practical to reprogram the Speaqualizer every time a departure from standard practice is encountered.

The Speaqualizer will be offered for sale by the American Printing House for the Blind, 1839 Frankfort Avenue, Louisville, KY 40206, perhaps in April 1987, for $\$ 950$. The software solutions to the screen review problem are somewhat less expensive than this. Blind computer operators have learned to expect that the hardware devices they must buy for use with their computers will usually cost thousands of dollars. The way in which a price of $\$ 950$ was accomplished deserves some discussion.

Hardware devices are usually expensive because the companies that sell them for profit must recover their development, production, and marketing costs. These companies can expect few sales for their products in comparison to the sales that can be expected for products sold in an unrestricted market, and if their operations are to be profitable, they must charge high prices for their products. Because they must do this, their customers are, for the most part, large companies, universities and school systems, state departments of rehabilitation, and government agencies. Most of the blind people who could make good use of their products will never have the chance to do so, because they will never be able to afford them. The free enterprise approach, although generally fruitful when manufacturers can compete for customers in an unrestricted market, is not an effective approach for delivering needed products to a restricted market such as the market constituted by blind computer operators.

Another approach was taken by the R\&D Committee of NFB, and this approach may well be worth emulating. The committee members who guided the development of the Speaqualizer donated their services and NFB assumed all other costs of development. The R\&D Committee did not terminate development when a successful prototype had been demonstrated. Instead, they carried development forward until they were in a position to deliver the procedures and tools for manufacturing the 
device. Thus they were able to spare a prospective manufacturer the costs of getting into production. Then they arranged for the manufacture and marketing of the Speaqualizer by the American Printing House for the Blind, a nonprofit company. The result is that a useful product, which might otherwise have cost as much as $\$ 3,000$, has been brought to market for only $\$ 950$-a price that, although not trivial, can be met by most blind computer operators who really want Speaqualizers.

Blind computer operators, armed with Speaqualizers and the ability to use them efficiently, can perform the tasks that make people worth employing in a wide variety of jobs. Furthermore, they can often be accommodated with little of the expense and alteration of office routine that typically attends the hiring of a printhandicapped person. IBM PCs dominate the business world, and wherever there are IBM PCs, PC XTs, PC ATs, or any of the personal computers that are compatible with these machines, blind workers can be accommodated simply by providing empty card slots for their Speaqualizers. They can use the same computers and, for the most part, the same application programs used by the sighted workers with whom they interact.

\section{REFERENCES}

Chong, C. (1986, January). Speech output for the IBM PCs. Braille Monitor. (Available from National Federation of the Blind, 1800 Johnson Street, Baltimore, MD 21230)

CROFT, D. L. (Ed.). (1984). A buyer's guide to computers for the blind and visually impaired. Boston, MA: National Braille Press.

CRofT, D. L. (Ed.). (1986). Add-ons: The ultimate guide to peripherals for the blind computer user. Boston, MA: National Braille Press.

EsPINOLA, E. (1985). A consumer's guide to technology for blind and sighted people. Sensus, 40, 1-25.

FouLKE, E. (1982). Microcomputers, VIPs, and the communication network. In J. Raviv (Ed.), Uses of computers in aiding the disabled (pp. 393-403). Amsterdam: North Holland Publishing.

LowRY, S. (1985). Speech resources. Houston, TX: Speech Enterprises.

McGillivray, R., \& Gast, M. (Eds.). (1983). Aids and appliances review: Voice output for computer access by the blind and visually impaired (Issue 9, Issue 10). Newton, MA: Carroll Center for the Blind.

Melrose, S. (1984). VersaBraille. In R. McGillivray \& M. Gast (Eds.), Aids and appliances review: Braille and computers (Issue 11, p. 14). Newton, MA: Carroll Center for the Blind.

SCAdDEN, L. (1983). Speech synthesizers. In R. McGillivray \& M. Gast (Eds.), Aids and appliances review: Voice output for computer access by the blind and visually impaired (Issue 9, Issue 10). Newton, MA: Carroll Center for the Blind. 